\title{
VALIDAÇÃO DA ESCALA DE COMPORTAMENTOS SOCIAIS DE PRÉ-ESCOLARES PARA O BRASIL ${ }^{1}$
}

\author{
Talita Pereira Dias* \\ Lucas Cordeiro Freitas \\ Zilda Aparecida Pereira Del Prette ${ }^{\mathfrak{\infty}}$ \\ Almir Del Prette ${ }^{\Phi}$
}

\begin{abstract}
RESUMO. Este estudo teve por objetivo verificar as qualidades psicométricas do instrumento norte-americano Preschool and Kindergarten Behavior Scale (PKBS) para o contexto brasileiro. Participaram, como informantes, pais e professores de 143 crianças de idade entre três e seis anos, de duas cidades do Interior do Estado de São Paulo. O PKBS-BR compõe-se de duas escalas, a saber, uma de habilidades sociais e uma de comportamentos problemáticos. Pais e professores responderam individualmente ao PKBS-BR na presença do aplicador. A análise fatorial indicou uma estrutura de três fatores para a escala de habilidades sociais (Cooperação Social, Independência Social e Interação Social) e dois fatores para a escala de comportamentos problemáticos (Externalizantes e Internalizantes), os quais explicaram, respectivamente, 45,38\% e 42,12\% da variância dos dados. Os indicadores de consistência interna foram satisfatórios para as escalas, variando de 0,79 a 0,95. Discutem-se algumas semelhanças e diferenças da escala original com a brasileira e as aplicações do PKBS para a pesquisa e intervenção psicológica.
\end{abstract}

Palavras-chave: Habilidades sociais; comportamentos problemáticos; qualidades psicométricas.

\section{VALIDATION OF THE PRESCHOOL AND KINDERGARTEN BEHAVIOR SCALES TO BRAZIL}

\begin{abstract}
This study aimed to verify the psychometric qualities of the Preschool and Kindergarten Behavior Scale $(P K B S)$ for the Brazilian context. Subjects were parents and teachers of 143 children, between three and six years old, from two inner cities of the São Paulo (Brazil). The PKBS-BR has two forms, one for social skills and another for behavior problems. Parents and teachers individually completed the PKBS-BR. Factor analysis indicated a three factor structure for the social skills scale (Social Cooperation, Social Independence and Social Interaction) and two factors for the behavior problems scale (Externalizing and Internalizing problems). The factors explained, respectively, $45.38 \%$ and $42.12 \%$ of the data variance. The internal consistency measures were satisfactory, varying between 0.79 and 0.95 . Some similarities and differences between American and Brazilian versions and the PKBS utility for research and psychological intervention are discussed.
\end{abstract}

Key words: Social skills; behavior problems; psychometric qualities.

\section{VALIDACIÓN DE LA ESCALA DE CONDUCTA SOCIAL PREESCOLAR EN BRASIL}

RESUMEN. Este estudio verificó las calidades psicométricas del Preschool and Kindergarten Behavior Scale (PKBS) para el contexto brasileño. Los participantes eran los padres y maestros de 143 niños, entre tres y seis años, de dos ciudades internas del Sao Paulo (Brasil). El PKBS-BR tiene dos formularios, uno para las habilidades sociales y otro para los problemas de conducta. Los padres y maestros le contestaron individualmente al PKBS-BR. Análisis de factor indicó una estructura de tres de factor para las habilidades sociales (Cooperación Social,

\footnotetext{
1 Apoio: FAPESP.

Mestre em Programa de Pós Graduação em Psicologia pela Universidade Federal de São Carlos, Brasil.

Il Doutor em Educação Especial (Educação do Indivíduo Especial) pela Universidade Federal de São Carlos, Brasil..

x Doutora em Psicologia (Psicologia Experimental) pela Universidade de São Paulo, com pós-doutorado em Psicologia das Habilidades Sociais na Universidade da Califórnia. Professora da Universidade Federal de São Carlos, Brasil.

$\Phi$ Doutor em Psicologia (Psicologia Experimental) pela Universidade de São Paulo.Professor da Universidade Federal de São Carlos , Brasil.
} 
Independencia Social y Interacción Social) y dos factores para los problemas de conducta descascaran (Externalizantes y Internalizantes). Los factores explicaron, respectivamente, $45.38 \%$ y $42.12 \%$ de la variación de los datos. Las medidas de consistencia interiores fueran satisfactorias, mientras variando entre $0.79 \mathrm{y} 0.95$. Se discute un poco de similitudes y diferencias entre la escala americana y la versión brasileña así como la utilidad del PKBS para la investigación y la intervención psicológica.

Palabras-clave: Las habilidades sociales; los problemas de conducta; las calidades psicométricas..

Conforme Del Prette e Del Prette (2001), dentre os vários conceitos do campo teóricoprático das habilidades sociais, três se revestem de maior importância, especialmente porque constituem a base para os demais. São eles: desempenho social, habilidades sociais e competência social. Para os autores, o desempenho social refere-se à totalidade de comportamentos sociais emitidos em interação social, enquanto as habilidades sociais consistem no conjunto de comportamentos sociais presentes no repertório do indivíduo que, se emitidos, contribuem para que um desempenho social seja qualificado como socialmente competente. A competência social é um termo de caráter avaliativo e implica na articulação entre pensamentos, sentimentos e comportamentos em função de objetivos pessoais e de demandas situacionais e culturais que podem produzir consequências favoráveis ao próprio indivíduo e à sua relação com os outros. Segundo Gresham e Elliott (1990), no caso de crianças, os critérios de competência social são o status social da criança entre os pares e julgamento positivo por outros significantes e outros comportamentos adaptativos, como o bom desempenho escolar e o autocuidado.

As classes de habilidades sociais mais importantes na infância, de acordo com Del Prette e Del Prette (2005a) são: autocontrole e expressividade emocional, habilidades de civilidade, empatia, assertividade, solução de problemas interpessoais, capacidade de fazer amizades e habilidades sociais acadêmicas. Há evidências de que um bom repertório de habilidades sociais na infância contribui para um bom rendimento acadêmico e para o ajustamento psicológico e social em etapas posteriores do desenvolvimento. Por outro lado, um repertório deficitário de habilidades sociais está associado a dificuldades de aprendizagem, problemas de comportamento, envolvimento com grupos de risco, isolamento social, delinquência e outros transtornos psicológicos como o comportamento antissocial, a fobia, a depressão e outros (Del Prette \& Del Prette, 2005a). Neste sentido, a promoção de habilidades sociais na infância pode contribuir para o bem-estar e adaptação da criança em curto, médio e longo prazo, ao mesmo tempo em que atua na prevenção ou atenuação de fatores de risco ao desenvolvimento de transtornos, entre eles, os problemas de comportamento.

Os problemas de comportamento estão relacionados a diversos transtornos e a déficits em habilidades sociais, por isso devem ser foco de investigação no tocante às habilidades sociais. Vários autores (Barreto, 2007; Del Prette \& Del Prette, 2005a; Elliott, Gresham \& Beddow, 2007; Gresham, 2009) enfatizam que déficits em habilidades sociais muita frequentes estão associados a problemas de comportamento que competem com a aquisição e ampliação do repertório de habilidades sociais. Além disso, estudos têm apontado que a intervenção precoce em crianças que apresentam problemas de comportamento é mais eficaz do que quando implementada em fases posteriores (Gresham, Lane, MacMillan \& Bocian, 1999; Loeber, 1991). Não obstante, para o planejamento e a implementação de intervenções que visem à promoção de habilidades sociais e à prevenção ou atenuação de problemas de comportamento na infância, há um pré-requisito importante: a avaliação confiável e válida desse repertório.

A avaliação de habilidades sociais vem sendo bastante discutida no campo das habilidades sociais (Curran, 1982; Del Prette, Monjas \& Caballo, 2006; Merrell, 1997), principalmente a partir de uma perspectiva multimodal, que se refere ao uso de diferentes informantes, procedimentos e/ou instrumentos de avaliação (Achenbach, McConaughy \& Howell, 1987). Na avaliação de habilidades sociais, a combinação de diferentes procedimentos e instrumentos é recomendada como forma de assegurar maior complementaridade e confiabilidade aos 
resultados obtidos (Del Prette \& Del Prette, 2005a; 2006), dada a especificidade das habilidades sociais e a multidimensionalidade desse conceito.

A especificidade das habilidades sociais é decorrente das expectativas do contexto social quanto aos comportamentos desejáveis dos indivíduos de acordo com o sexo, idade, papel social, a condição orgânica, entre outros (Del Prette \& Del Prette, 2005a) de modo que, por exemplo, os comportamentos valorizados para uma criança de quatro anos podem ser bem distintos dos que são valorizados para uma de seis. Adicionalmente, a cultura e a subcultura podem valorizar o ensino de outras classes de habilidades sociais em função do sexo da criança. O estudo de Garcia-Serpa, Del Prette e Del Prette (2006) traz evidências de maior valorização das habilidades sociais empáticas em meninas do que em meninos.

Diferentes critérios de avaliação do desempenho social das crianças podem variar também em função de diferentes contextos, como o escolar e o familiar. Estudos mostram que professoras de pré-escolares com problemas de comportamento, quando comparadas às mães dessas crianças, relatam em suas avaliações maior frequência de comportamentos problemáticos e menor frequência de habilidades sociais (BolsoniSilva, Marturano, Pereira \& Manfrinato, 2006).

A especificidade das habilidades sociais esperadas para os diferentes momentos do desenvolvimento e para os diferentes contextos e interlocutores indica a necessidade e importância de estudos de levantamento e de normatização para diferentes faixas etárias na infância. No Brasil, alguns estudos foram conduzidos nessa perspectiva - como, por exemplo, os efetuados na validação do Inventário Multimídia de Habilidades Sociais para Crianças (Del Prette \& Del Prette, 2005b) e do Sistema de Avaliação de Habilidades Sociais (Bandeira, Del Prette, Del Prette \& Magalhães, 2009). Não obstante, ambos os estudos foram realizados com amostras de crianças a partir de sete anos já inseridas no primeiro e segundo ciclos do Ensino Fundamental, sendo escassos os estudos com a população préescolar.

Recentemente, foi publicado estudo sobre a validação de um instrumento de avaliação de habilidades sociais para pré-escolares (BolsoniSilva, Marturano \& Loureiro, 2009). Esse instrumento, denominado Questionário de Respostas Socialmente Habilidosas para professores (QRSH-PR), constitui-se de 24 itens de avaliação dos professores quanto às habilidades sociais de seus alunos pré-escolares. Os resultados indicaram propriedades psicométricas satisfatórias em termos de consistência interna, validade de construto, preditiva, concorrente e discriminativa; no entanto esse instrumento foi validado somente na versão dos professores e se restringe à avaliação de habilidades sociais sem incluir os comportamentos problemáticos.

Defende-se que a avaliação concomitante de comportamentos problemáticos é relevante, pois estes concorrem com a emissão de habilidades sociais (Gresham 2009) e devem, em muitos casos, ser alvo de intervenção de programas de habilidades sociais. Outro aspecto considerado de importância é a avaliação dos pais, uma vez que estes são os primeiros agentes da socialização das crianças e observadores privilegiados de comportamentos sociais em vários contextos (família, vizinhança, shoppings, etc.), podendo confirmar ou não a generalidade dos comportamentos que ocorrem na escola.

O PKBS-BR (Preschool Kingarten Behavior Scales- PKBS), um instrumento norte-americano de avaliação de habilidades sociais e comportamentos problemáticos em pré-escolares para pais e professores desenvolvido por Merrell (2002), foi traduzido para a língua portuguesa por Del Prette e Del Prette (2006) e disponibilizado em espanhol por Del Prette et al. (2006). Esse instrumento vem sendo utilizado em pesquisas nos Estados Unidos (Matson \& Wilkins, 2009), conta com uma versão validada para a comunidade hispânica (Carney \& Merrell, 2002) e foi adaptado também em outros países, como, por exemplo, a Argentina (Reyna \& Brussino, 2009).

Tendo-se em vista a importância da avaliação multimodal por meio da adoção de diferentes informantes, como pais e professores, e da intervenção precoce no repertório de habilidades sociais e problemas de comportamento da criança para atenuar riscos e promover ajustamento psicossocial, o presente estudo teve como objetivo verificar as qualidades psicométricas, especificamente a consistência interna e a validade de construto do PKBS-BR como parte do processo de adaptação desse instrumento no contexto brasileiro. 


\section{MÉTODO}

O projeto mais amplo do qual este trabalho faz parte foi aprovado pelo Comitê de ética em pesquisas com seres humanos (CEP) da Universidade Federal de São Carlos, sob o número 330/2008.

\section{Participantes}

Participaram como informantes 143 mães e vinte e duas professoras de um grupo de 143 crianças ( 80 meninas e 63 meninos) de idade entre três e seis anos e idade média de 4,8 anos $(\mathrm{dp}=$ $0,79)$. Todas as crianças foram avaliadas pelas mães e pelas professoras. As crianças eram provenientes de duas escolas públicas de Educação Infantil e de uma creche assistencial localizadas em duas cidades do Interior do Estado de São Paulo. Uma escola situa-se em área central e as outras instituições estão localizadas em bairros periféricos. A distribuição quanto ao nível socioeconômico das famílias das crianças foi: A1 $(1 \%)$, A2 $(2 \%)$, B $1(8 \%)$, B2 $(15 \%)$, C $(50 \%)$, D $(23 \%)$ e E $(1 \%)$.

\section{Instrumento}

A Escala de Comportamentos Sociais de Préescolares (PKBS-BR) foi produzida originalmente nos EUA (Preschool Kindergarten Behavior Scales - 2, Merrell, 2002) e é amplamente utilizada naquele país para a avaliação de habilidades sociais e comportamentos problemáticos de pré-escolares. Suas qualidades psicométricas foram verificadas, tendo-se constatado uma consistência interna de $\alpha=0.96$ para a escala de habilidades sociais e de $\alpha=0.97$ para a de comportamentos problemáticos, considerando-se a amostra total (pais e professores) (Merrell, 2002). A estrutura fatorial na versão estadunidense se compõe de três fatores na escala de habilidades sociais: Cooperação Social $(\alpha=0.94)$; Interação Social $(\alpha=0.92)$; Independência Social $(\alpha=0.88)$. Os itens da escala de comportamentos problemáticos agruparam-se em dois fatores: problemas externalizantes $(\alpha=0.97)$ e problemas internalizantes $(\alpha=0.90)$. Esta escala, ainda não validada para o contexto brasileiro, é apresentada em uma versão que pode ser respondida tanto por pais como por professores, e contém trinta e quatro itens de habilidades sociais e quarenta e dois itens de comportamentos problemáticos. Os itens são avaliados em termos de frequência em uma escala do tipo Likert, de quatro pontos: $0=$ nunca; $1=$ raramente; $2=$ algumas vezes; $3=$ frequentemente. $\mathrm{Na}$ versão brasileira, além da avaliação da frequência das habilidades sociais e dos comportamentos problemáticos, é também avaliada sua importância, a qual é atribuída em uma escala do tipo Likert, de três pontos: $0=$ nenhuma importância; $1=$ alguma importância; e $2=$ muita importância. Essa avaliação é feita por adultos significativos para o desenvolvimento da criança e objetiva verificar a validade social dos itens de habilidades sociais.

\section{Procedimento de coleta de dados}

A aplicação dos instrumentos foi feita por um dos pesquisadores nas próprias instituições frequentadas pelas crianças. Os objetivos do estudo e a forma de participação foram apresentados aos pais e professores, destacando-se que não existiam respostas certas ou erradas e que era necessário responderem a todos os itens. Os questionários foram respondidos individualmente pelos pais e professores em presença da pesquisadora.

No caso da aplicação aos pais, para aqueles que relataram ou sinalizaram algum tipo de dificuldade no entendimento dos itens ou no preenchimento do questionário, a pesquisadora leu os itens e preencheu no formulário as respostas dadas por eles. Os exemplos para melhor compreensão do item foram sempre os mesmos para todos os pais que requereram ajuda. Cada professor respondeu a um questionário referente a um de seus alunos, e o fez na presença da pesquisadora, a qual ofereceu instruções padronizadas e esclareceu dúvidas a respeito dos itens e do preenchimento, assim como procedeu na aplicação aos pais. Foram disponibilizados aos professores os demais questionários que eles deveriam preencher no caso de terem mais alunos, e eles tiveram vinte e cinco dias para devolvê-los à pesquisadora.

Foram respondidas duas escalas para cada criança participante, a saber, uma foi respondida pelos pais e a outra pela professora, totalizando 286 escalas preenchidas. A abordagem multimodal neste estudo caracterizou-se pela adoção de pais e professores como informantes e uso de indicadores de habilidades sociais e comportamentos problemáticos, os quais são vistos como comportamentos concorrentes das habilidades sociais (Gresham, 2009). 


\section{Procedimento de tratamento de dados}

Os dados obtidos com base na avaliação das mães e das professoras de cada criança com o PKBS-BR foram computados sob a forma de escores, de acordo com instruções do manual, e organizados em planilhas do SPSS 16.0. Somente foram utilizados os dados de crianças que tanto as mães quanto as professoras haviam avaliado. Análises estatísticas com testes pertinentes aos objetivos específicos foram efetuadas, adotandose o nível de significância de $0,05(p<0,05)$.

Para a verificação da validade de construto do instrumento foi realizada uma análise fatorial exploratória, tanto da escala de habilidades sociais como da de comportamentos problemáticos. A confiabilidade em termos de consistência interna foi baseada no Coeficiente Alfa de Cronbach. Nessas análises foram utilizados conjuntamente os dados obtidos por meio da avaliação dos pais e aqueles obtidos pela avaliação das professoras, seguindo-se o mesmo procedimento adotado pelo autor da escala norte-americana (Merrell, 2002).

\section{RESULTADOS}

A seguir serão apresentados os resultados do estudo, divididos em duas etapas: (1) análise fatorial exploratória do instrumento e (2) consistência interna, por meio do coeficiente Alfa de Cronbach.

\section{Análise fatorial}

Para se verificar a estrutura fatorial do PKBS$\mathrm{BR}$, foi realizada uma análise fatorial exploratória pelo método dos Componentes Principais com rotação Varimax. Foram realizadas diferentes alternativas de delimitação da quantidade de fatores, tendo-se como base: (a) a restrição de saturação dos itens de pelo menos 0,40 ; (b) o valor dos autovalores superior a 1,00; e (c) a interpretabilidade teórica do agrupamento dos itens. Após sucessivas análises dos componentes principais realizadas de acordo com esses critérios, chegou-se a uma estrutura interpretável de três fatores para a escala de habilidades sociais e dois fatores para a escala de comportamentos problemáticos.

A estrutura fatorial das escalas de habilidades sociais e de comportamentos problemáticos, com os coeficientes de saturação dos itens em cada fator, pode ser observada na tabela 1 .

Tabela 1. Estrutura fatorial das escalas de avaliação de habilidades sociais e comportamentos problemáticos, com os coeficientes de saturação dos itens em cada fator.

\begin{tabular}{cccccccc}
\hline \multicolumn{3}{c}{ Habilidades Sociais } & & & \multicolumn{3}{c}{ Comportamentos } \\
Problemáticos
\end{tabular}

\section{Escala de Habilidades Sociais.}

A análise fatorial apontou que esta escala possui uma estrutura com trinta e dois itens, distribuídos em três fatores, com autovalores entre 10,00 e 1,94, que 
explicam 45,38\% da variância dos dados. A medida de adequação da amostra (K.M.O.=0,90) foi satisfatória e o teste de Bartlet foi significativo $\left(\chi^{2}=4013,85\right.$; $\mathrm{df}=561$; e $\mathrm{p}=0,00)$. Os itens desta escala obtiveram coeficientes de saturação entre 0,40 e 0,83 , sendo que os itens 6, 11, 21 e 34 se encontraram em mais de um fator. Os itens 13 e 30 foram eliminados da estrutura fatorial, pois obtiveram cargas fatoriais inferiores a 0,40. Os fatores identificados foram assim denominados: F1 - Cooperação Social (13 itens), F2 Independência Social (11 itens) e F3 - Interação Social (8 itens).

\section{Escala de Comportamentos Problemáticos.}

A análise fatorial indicou que esta escala possui uma estrutura com quarenta e dois itens, distribuídos em dois fatores, com autovalores entre 14,62 e 3,07, que explicaram $42,12 \%$ da variância dos dados. A medida de adequação da amostra (K.M.O.=0,93) foi satisfatória e o teste de Bartlet foi significativo $\left(\chi^{2}=5642,13 ; \mathrm{df}=861\right.$ e $\left.\mathrm{p}=0,00\right)$. Os itens desta escala obtiveram coeficientes de saturação entre 0,43 e 0,77 , sendo que os itens $7,10,18,35$ e 41 foram encontrados em mais de um fator. Nenhum item foi eliminado da estrutura fatorial, pois todos obtiveram cargas fatoriais superiores a 0,40 . Os fatores identificados foram assim denominados: F1 Comportamentos Externalizantes (24 itens), F2 Comportamentos Internalizantes (18 itens).

\section{Consistência Interna}

Com relação à consistência interna, tanto para a escala de comportamentos problemáticos como para a escala de habilidades sociais, os índices foram considerados adequados, como se pode ver na Tabela 2.

Tabela 2. Indicadores de consistência interna do PKBS-BR para cada escala e fatores.

\begin{tabular}{ccc}
\hline Escalas e Fatores & Alfa de Cronbach & Número de Itens \\
\hline Escala de Habilidades Sociais & $\alpha=0,92$ & 32 \\
F1 - Cooperação Social & $\alpha=0,89$ & 13 \\
F2 - Independência Social & $\alpha=0,86$ & 11 \\
F3 - Interação Social & $\alpha=0,79$ & 8 \\
Escala de Comportamentos problemáticos & $\alpha=0,95$ & 42 \\
F1- Comportamentos Externalizantes & $\alpha=0,95$ & 24 \\
F2 - Comportamentos Internalizantes & $\alpha=0,88$ & 18 \\
\hline
\end{tabular}

Com base na Tabela 2, pode-se observar que os coeficientes de consistência interna variaram de 0,79 (F3 - Interação Social) à 0,95 (Escala global de Comportamentos Problemáticos e F1 Comportamentos externalizantes).

\section{DISCUSSÃO E CONCLUSÃO}

Todos os valores de consistência interna obtidos para as escalas globais e para os fatores das escalas de habilidades sociais e de comportamentos problemáticos foram adequados e satisfatórios, de acordo com Bisquerra, Sarriera e Martinez (2004), pois estiveram acima de 0,75. Os coeficientes superiores a 0,75 para as escalas globais e os fatores indicaram que os itens do instrumento foram consistentes para avaliar diferentes classes de habilidades sociais e de comportamentos problemáticos, pois forneceram um indicador de confiabilidade ou fidedignidade à versão brasileira do instrumento, com base na homogeneidade dos seus itens; entretanto, na comparação da consistência interna entre as versões brasileira, norte-americana e espanhola, pode-se notar que os valores de alfa obtidos nesta análise foram mais baixos do que os encontrados nos outros estudos (Merrel, 2002; Muñoz et al., 2011), o que indica uma menor precisão da versão desse instrumento aqui apresentado.

Os fatores apontados pela análise fatorial no presente estudo foram semelhantes aos encontrados por Merrell (2002) no estudo de validação original, bem como no estudo de validação para a população espanhola (Muñoz et al., 2011). Na análise da versão original do instrumento, foram encontrados os mesmos três fatores de habilidades sociais identificados no presentes estudo: Cooperação Social, Interação Social e Independência Social (Merrell, 2002).

Comparando-se os fatores da versão norteamericana com aqueles obtidos na versão 
brasileira, pode-se notar que o fator 1 da versão original teve dez dos seus doze itens iguais aos do fator 1 da versão brasileira. Nove dos onze itens que compuseram o fator 2 da versão original foram os mesmos que os do fator 3 da versão brasileira. Por fim, o fator 3 continha oito itens que também compuseram o fator 2 da adaptação brasileira. Essas semelhanças igualmente foram verificadas na comparaçãos com a análise fatorial espanhola realizada no estudo de Muñoz et al. (2011). Além disso, foi observada uma inversão quanto à ordem dos agrupamentos dos itens: o fator 2 das versões original e espanhola apareceu como fator 3 na escala brasileira, enquanto o fator 3 das versões original e espanhola apareceu como fator 2 na escala brasileira. Esse resultado sugere uma diferenciação em termos da contribuição relativa ou do peso de cada fator nas diferentes populações para as quais o instrumento foi validado.

Quanto à escala de comportamentos problemáticos, a versão brasileira encontrou os mesmos dois fatores do instrumento original: Comportamentos Internalizantes

e Comportamentos Externalizantes (Merrell, 2002); mas na versão norte-americana o autor realizou uma nova análise fatorial, separada para cada um dos fatores encontrados (Comportamentos Internalizantes e Externalizantes), para verificar a existência de mais fatores específicos nessas dimensões. Como resultado, foram encontradas novas subescalas suplementares para cada tipo de problema de comportamento. Para Comportamentos Externalizantes foram encontradas três subescalas (Egocêntrico/Explosivo, Problemas de Atenção/Hiperativo e Antissocial/Agressivo) e para Comportamentos Internalizantes foram extraídas duas subescalas (Retraimento social e Ansiedade/Problemas Somáticos).

As diferenças na estrutura interna das versões norte-americana e brasileira do instrumento podem refletir diferenças culturais presentes na validação de escalas. No tocante às dimensões de habilidades sociais, a cultural envolve as regras, normas e costumes presentes em uma dada cultura que influenciam as relações interpessoais. Dessa forma, comportamentos valorizados e, em geral, frequentemente emitidos por membros de uma dada cultura podem ser diferentes daqueles emitidos por pessoas de outras culturas. Os comportamentos valorizados, tolerados ou punidos nos diferentes contextos culturais justificam a importância da validação de instrumentos de avaliação, estando em conformidade com o objetivo do presente estudo.

$\mathrm{O}$ desenvolvimento do PKBS-BR em termos de investigação das qualidades psicométricas, foco deste estudo, poderá viabilizar investigações que relacionem as habilidades sociais e problemas de comportamento em crianças pré-escolares. A avaliação de comportamentos sociais em crianças pequenas é amplamente defendida, ao se considerarem dados de estudos anteriores (Gresham, Lane, MacMillan \& Bocian, 1999; Loeber, 1991), os quais apontam para a establidade dos problemas de comportamento e seu agravamento ao longo do tempo, sugerindo que intervenções precoces têm maior possibilidade de ser efetivas. Apesar da evidente importância dos instrumentos de avaliação, no contexto brasileiro, estudos indicam a escassez de procedimentos e instrumentos de coleta de dados para a população pré-escolar (por exemplo, Löhr, 2003). Neste sentido, são necessários novos estudos visando tanto ao aperfeiçoamento de investigações com o PKBS-BR como ao desenvolvimento de outros instrumentos direcionados à população pré-escolar.

Não obstante, deve-se ressaltar que a amostra de crianças avaliadas neste estudo é ainda restrita tanto por seu tamanho $(\mathrm{N}=143)$ como por ser predominantemente de uma cidade do interior do Estado de São Paulo. Neste contexto, o presente estudo constitui uma validação exploratória e preliminar que deve ser aperfeiçoada com amostras mais abrangentes e diversificadas em novas pesquisas.

$\mathrm{O}$ PKBS-BR, enquanto instrumento de avaliação com qualidades psicométricas testadas, pode gerar uma avaliação mais precisa e válida dos déficits e dos recursos presentes no repertório comportamental da criança. Avaliações multimodais podem trazer desdobramentos para intervenções nos contextos escolar e clínico e, assim, oferecer subsídios para o planejamento de programas de intervenção e prevenção para a população pré-escolar. Ademais, intervenções pautadas nas necessidades próprias de cada criança ou grupo de crianças têm maior probabilidade de ser efetivas e reduzir a relação 
custo-benefício desse tipo de investimento em políticas públicas de atendimento à infância.

\section{REFERÊNCIAS}

Achenbach, T. M., McConaughy, S. H., \& Howell, C.T. (1987). Child/adolescent behavioral and emotional problems: implications of cross-informant correlations for situational specificity. Psychological Bulletin, 101(2), 213-32.

Bandeira, M., Del Prette, Z. A. P., Del Prette, A.; \& Magalhães, T. (2009). Sistema de Avaliação de Habilidades Sociais, SSRS-BR: Validação transcultural para o Brasil. Psicologia: Teoria $e$ Pesquisa, 25(2), 271-282.

Barreto, S.O. (2007). Habilidades sociais na comorbidade entre dificuldade de aprendizagem $e$ problemas de comportamento: uma avaliação multimodal. Dissertação de Mestrado Não-Publicada, Programa de Pós-Graduação em Educação Especial, Universidade Federal de São Carlos. São Carlos, SP.

Bisquerra, R., Sarriera, J. C., \& Martinez, F. (2004). Introdução à estatística: enfoque informático com o pacote estatístico SPSS. Porto Alegre: Artmed.

Bolsoni-Silva, A. T, Marturano, E. M., Pereira, V. A., \& Manfrinato, J. W. S (2006). Habilidades sociais e problemas de comportamento de pré-escolares: comparando avaliações de mães e de professoras. Psicologia: Reflexão e Crítica, 19(3), 460-9.

Bolsoni-Silva, A. T., Maturano, E. M., \& Loureiro, S. R. (2009). Construction and validation of the brazilian Questionário de Respostas Socialmente Habilidosas segundo relato de professores (QRSH-PR). Spanish Journal of Psychology, 12 (1), 349-59.

Carney. A. G., \& Merrell, K.W. (2002). Reliability and comparability of a Spanish-language form of the preschool and kindergarten behavior scales. Psychology in the schools, 39, 367-73.

Curran, J. (1982). A procedure for the assessment of social skills: The simulated social interaction. In J. Curran \& P.M. Monti (Eds.), Social skills training (pp. 348-73). New York: TGP.

Del Prette, A., \& Del Prette, Z. A. P. (2001). Psicologia das relações interpessoais: vivências para o trabalho em grupo. Petrópolis: Vozes.

Del Prette, Z. A. P. \& Del Prette, A. (2005b): Sistema Multimídia de Habilidades Sociais para Crianças (SMHSC-Del-Prette): Manual. São Paulo: Casa do Psicólogo (Inclui Cd-Rom e outros materiais impressos).

Del Prette, Z. A. P., \& Del Prette, A. (2005a). Psicologia das Habilidades Sociais na Infância: Teoria e Prática. Rio de Janeiro: Vozes.

Del Prette, Z. A. P., \& Del Prette, A. (2006). Avaliação multimodal de habilidades sociais em crianças: procedimentos, instrumentos e indicadores. In $\mathrm{M}$. Bandeira, Z.A.P. Del Prette \& A. Del Prette (Orgs.), Estudos sobre Habilidades Sociais e Relacionamento Interpessoal (pp. 47-68). São Paulo: Casa do Psicólogo.

Del Prette, Z. A. P., Monjas-Casares, M. I., \& Caballo, V. E. (2006). La evaluación del repertorio de las habilidades sociales en ninõs. In V.E Caballo (Org.), Manual para la evaluación clínica de los transtornos psicológicos: Transtornos de la edad adulta e informes psicológicos (pp.371-399). Madrid: Pirámide

Elliott, S. N., Gresham, F. M., Frank, J., \& Beddow, P. A. (2007). Intervention Validity of social behavior rating scales: features of assessments that link results to treatment plans. Manuscrito encaminhado pelo autor.

Garcia-Serpa, F. A., Del Prette, Z. A. P., \& Del Prette, A. (2006). Meninos pré-escolares empáticos e nãoempáticos: empatia e procedimentos educativos dos pais. Revista Interamericana de Psicologia, 40 (1), 77-88.

Gresham, F. M. (2009). Análise do Comportamento Aplicada às Habilidades Sociais. In A. Del Prette \& Z.A.P. Del Prette (Orgs), Psicologia das Habilidades Sociais: diversidade teórica e suas implicações (pp.17-66). Petrópolis, RJ: Vozes.

Gresham, F. M., \& Elliott, S. (1990). Social Skills Rating System. Circle Pines, MN: American Guidance Service.

Gresham, F. M., Lane, K. L., MacMillan, D. L., \& Bocian, K.M. (1999). Social and academic profiles of externalizing and internalizing groups: risk factors for emotional and behavioral disorders. Behavioral Disorders, 24 (3), p. 231-45.

Loeber, R. (1991). Antisocial behavior: more enduring than changeable? Journal of American Academy of Child and Adolescent Psychiatry, 30, p. 393-97.

Löhr, S. S. (2003). Estimulando o desenvolvimento de habilidades sociais em idade escolar. In A. Del Prette \& Z. Del Prette (Orgs), Habilidades sociais, desenvolvimento e aprendizagem: questões conceituais, avaliação e intervenção (pp. 293-310) Campinas, SP: Alínea.

Matson, J. L., \& Wilkins, J. (2009). Psychometric testing methods for children's social skills. Research in Developmental Disabilities, 30 (2), 249-74.

Merrell, K. W. (1997). Assessing social skills and peer relations. In H. B. Vance (Ed.), Psychological assessment of children: Best practices for school and clinical settings (2 ed., pp. 246-276). New York: John Wiley \& Sons.

Merrell. K. W. (2002). Preschool and Kindergarten Behavior Scales. 2 nd Ed. Austin, TX: PRO-ED

Muñoz, J. L. B., Martínez, M. C. P., Berbén, T. G., Cabezas, Justicia, M. F., F. J., \& de Haro, E. F. (2011). Análisis de la estructura factorial de las puntuaciones de la Preschool and Kindergarten Behavior Scale en población española. Psicothema 23, (2), 314-21. 
Reyna, C.E., \& Brussino, S.A. (2009) Propiedades psicométricas de la escala de comportamiento preescolar y jardín infantil (versión argentina abreviada). Anais do Seminário Internacional de Habilidades Sociais, 2 (p. 102). Rio de Janeiro: Universidade do Estado do Rio de Janeiro.

\section{Endereço para correspondência:}

Talita Pereira Dias. Programa de Pós Graduação em Psicologia, Centro de Educação e Ciências Humanas. Rodovia Washington Luis, km 235, Bairro Monjolinho, CEP 13565-905, São Carlos-SP, Brasil. E-mail: talitapsi10@yahoo.com.br. 